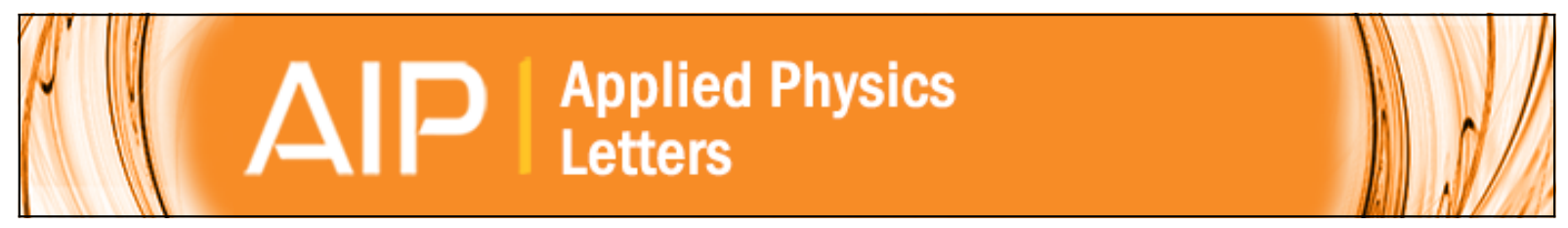

\title{
Measurement of the phase shift upon reflection from photonic crystals
}

Emanuel Istrate and Edward $\mathrm{H}$. Sargent

Citation: Applied Physics Letters 86, 151112 (2005); doi: 10.1063/1.1905812

View online: http://dx.doi.org/10.1063/1.1905812

View Table of Contents: http://scitation.aip.org/content/aip/journal/apl/86/15?ver=pdfcov

Published by the AIP Publishing

\section{Articles you may be interested in}

Polarization sensitive near-complete reflection from photonic crystal slab in centered rectangular lattice J. Appl. Phys. 110, 023103 (2011); 10.1063/1.3610518

Phase properties of reflected light in photonic band gap J. Appl. Phys. 107, 093108 (2010); 10.1063/1.3383045

Enhanced Bragg reflections from size-matched heterostructure photonic crystal thin films prepared by the Langmuir-Blodgett method

Appl. Phys. Lett. 89, 093116 (2006); 10.1063/1.2339031

Optical emission from individual InGaAs quantum dots in single-defect photonic crystal nanocavity

J. Appl. Phys. 98, 034306 (2005); 10.1063/1.1953885

Compound cavity measurement of transmission and reflection of a tapered single-line photonic-crystal waveguide

Appl. Phys. Lett. 82, 2577 (2003); 10.1063/1.1568821

\section{AIP $\left.\right|_{\text {APL Photonics }}$}

APL Photonics is pleased to announce Benjamin Eggleton as its Editor-in-Chief

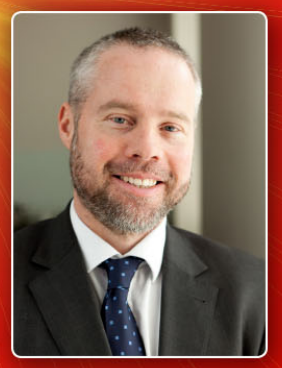




\title{
Measurement of the phase shift upon reflection from photonic crystals
}

\author{
Emanuel Istrate ${ }^{a)}$ and Edward H. Sargent \\ Department of Electrical and Computer Engineering, University of Toronto, 10 King's College Road, \\ Toronto, Ontario M5S 3G4, Canada
}

(Received 10 August 2004; accepted 3 March 2005; published online 7 April 2005)

\begin{abstract}
The phase dependence of light reflected from colloidal photonic crystals is measured using a large resonant cavity and self-assembled colloidal photonic crystals. We measure the expected phase shift upon reflection from the photonic crystal, which varies from 0 to $180 \mathrm{deg}$ across the photonic crystal stop band. These measurements are then fed directly into the design of photonic crystal cavities. We obtain a measure for the precision needed in the fabrication of photonic crystal resonant cavities. () 2005 American Institute of Physics. [DOI: 10.1063/1.1905812]
\end{abstract}

The reflection and transmission characteristics of photonic crystals are among their most important properties for practical devices, such as waveguides and optical filters. They have been measured thoroughly in many types of photonic crystals, both two and three dimensional. At optical frequencies, however, most measurements have focused on the intensity of transmission and reflection. ${ }^{1,2}$ This can be used to obtain the amplitude of the reflection and transmission coefficients.

Photonic crystals are used as reflectors in a variety of devices, such as waveguides or resonant cavities. The reflection properties are especially important in cavity resonators, where improvements in the crystal configuration near the cavity have produced quality factors of several tens of thousands. ${ }^{3,4}$ Low-loss waveguides have also been demonstrated. ${ }^{5}$ While the amplitude of reflection and the spatial frequencies in the cavity determine the loss of power from the resonator and the quality factor, the phase change upon reflection will determine the resonant frequencies. ${ }^{6} \mathrm{Re}-$ flection from a photonic crystal stop band produces a phase change between $0^{\circ}$ and $180^{\circ}$. Its exact value needs to be known in order to design suitable microcavities and to understand the resonances which are observed. Resonant modes in cavities have been described approximately based on device symmetry. ${ }^{7}$ Knowledge of the reflection phase would make this method exact. In this letter we report measurements of the phase of reflected waves from a photonic crystal.

At microwave frequencies both the reflection amplitude and phase have been measured. ${ }^{8}$ The phase evolution of light inside periodic structures has been measured using a tapered fiber to extract the evanescent fields above the structure. ${ }^{9}$ This provides information on the phase velocity of light in the crystal. The standing waves observed in front of the crystal can be used to extract the reflection phase. This can be done, however, only with crystals in special slab waveguides that have a large evanescent tail in air, and requires a complex setup. Furthermore, since the phase is extracted from the standing-wave intensity patterns, the sign of the phase shift is lost.

Light reflected from a dielectric interface below the critical angle is either in phase or $180 \mathrm{deg}$ out of phase with the incident wave, giving us a real reflection coefficient. As a

\footnotetext{
a) Author to whom correspondence should be addressed; electronic mail:
} e.istrate@utoronto.ca result, Fabry-Pérot cavities made with such interfaces as reflectors have a length equal to an integer number of halfwavelengths at resonance. On the other hand, total internal reflection from a dielectric interface produces a complex reflection coefficient. The reflection phase can take any value. This is the reason why the width of the core of a slab waveguide is not equal to an integer number of transverse wavelengths. Light reflected by a photonic crystal stop band receives a phase shift, similar to that from total internal reflection, which affects the required width of photonic crystal waveguide cores or resonant cavities in the same way.

Photonic crystals exhibit various forms of disorder, or deviations from perfect periodicity, ${ }^{10-12}$ which can change their optical properties. For this reason it is important to have as direct as possible a measure of the phase upon reflection for use in device design.

In order to measure the reflection phase, we employ a very wide Fabry-Pérot cavity, using the photonic crystal as one of the reflectors. We use colloidal self-assembled polystyrene opals grown by convective assembly from a water solution. ${ }^{13}$ The sphere diameter is $450 \mathrm{~nm}$ and their index of refraction is 1.6, giving us a stop band in the (111) direction at approximately $1100 \mathrm{~nm}$. We use a gold reflector on the other side of the cavity, to obtain a high reflection, similar in magnitude to that of the photonic crystal. The structure of the sample is shown in Fig. 1. The cavity is made of glass, with a length, $L$, of $155 \mu \mathrm{m}$. The photonic crystal is approximately 30 layers thick, and we use $30 \mathrm{~nm}$ of gold.

The phase change during reflection can be interpreted as a penetration into the crystal, which affects the resonant frequencies of the cavity. We compare these frequencies with

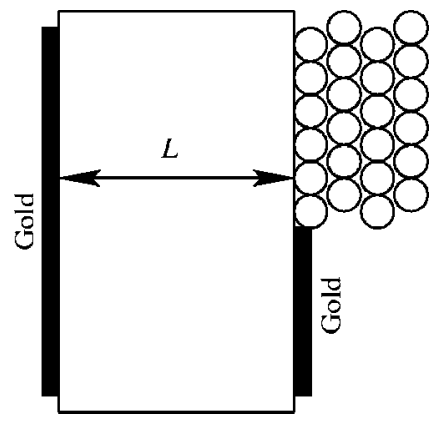

FIG. 1. Resonant cavity used to measure the reflection phase. The reflection phase can be interpreted as a penetration into the crystal, resulting in a change in the resonance wavelengths. Lower part is used as a reference. 


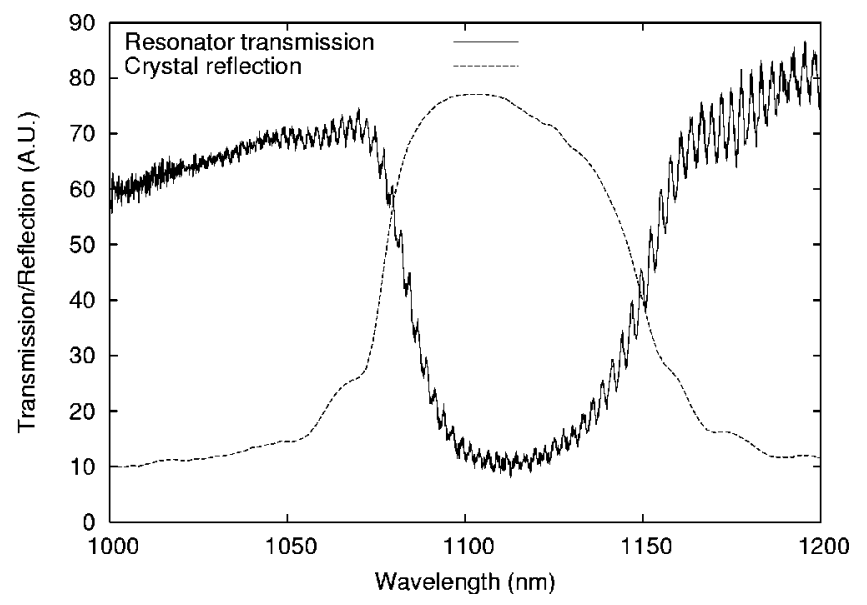

FIG. 2. Transmittance of the photonic crystal resonator. The fast oscillations are due to the wide resonant cavity. The reflectance of a single photonic crystal is also shown.

those from an area of the sample without the photonic crystal, shown in the lower part of Fig. 1. Our wide cavity produces 35 resonant transmittance peaks in the stop band. This allows us to obtain a very detailed measurement of the phase throughout the stop band. In Fig. 2 we show the transmittance spectrum of the resonator, as well as the reflectance of the photonic crystal.

Figure 3 compares the peaks of the resonator with one gold and one photonic crystal reflector with those of a resonator with gold reflectors on both sides. The two plots cover wavelengths near the two edges of the stop band, and show that the reflection phase from the photonic crystal is the same as from gold at the long-wavelength edge of the stop band, but is $180 \mathrm{deg}$ out of phase at the other edge. Throughout the stop band the phase changes smoothly. In other words, the reflection at the long-wavelength stop band edge is similar to that from a perfect electric conductor, while at the other band edge it behaves like that from a perfect magnetic conductor.

To calculate the phase change from the measured resonances we extend the usual relation for the resonant peaks of the Fabry-Pérot interferometer to include the phase change as follows:
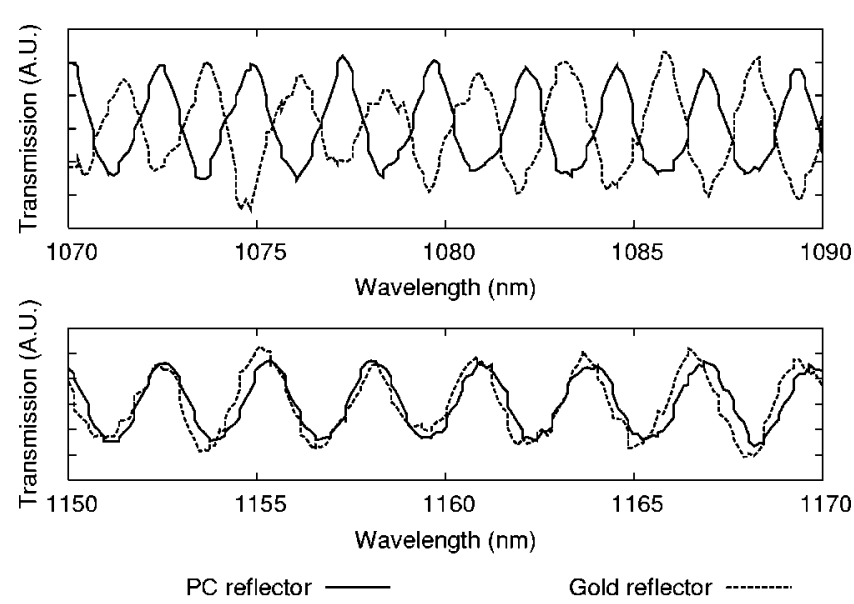

FIG. 3. Comparison of resonant peaks for cavities with and without a photonic crystal reflector. At the lower stop band edge the reflection from the photonic crystal is out of phase with that from the gold reflector At the other edge they are in phase.

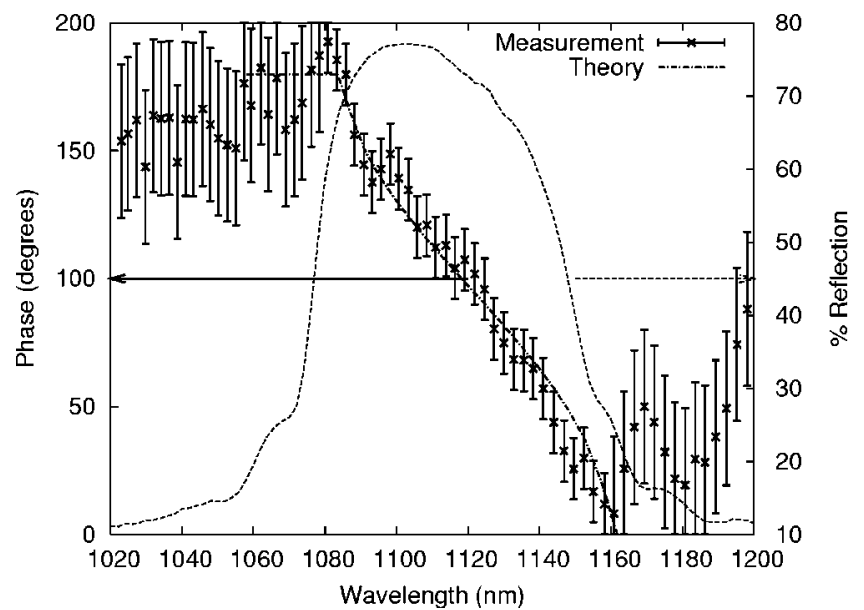

FIG. 4. Phase change (measurement and theory) for reflection from a photonic crystal, showing the change from zero to 180 deg across the stop band. Single crystal reflectance also shown for reference.

$$
2 \frac{2 \pi n}{\lambda} L+\phi=2 m \pi
$$

where $\phi$ is the phase change at one of the interfaces, $n$ is the index of refraction of the cavity, $\lambda$ is the vacuum wavelength and $m$ is an integer. From this we obtain

$$
\phi=\Delta f \frac{4 \pi n L}{c},
$$

where $\Delta f$ is the difference in resonant frequency between the sample with and without the photonic crystal and $c$ is the speed of light. $n L$ can be calculated from the distance between transmission peaks of the resonator.

Figure 4 shows the measured phase change for our colloidal photonic crystals, along with the intensity reflection spectrum of a single photonic crystal, which shows the position of the stopband. There is a difference of $180^{\circ}$ between the reflection phases on the two sides of the stop band, as described previously. The plot also shows that the phase change varies smoothly across the stopband, taking the value of $90^{\circ}$ near the center of the stop band. In Fig. 4 we also plot the result expected from a theoretical analysis, done by enforcing the electromagnetic boundary conditions between plane waves in glass and the decaying stopband modes of the photonic crystal. ${ }^{6}$ Good agreement is obtained with our measurements.

Outside the stop band reflection properties are similar to those of a homogeneous dielectric. No phase change is expected. Measurements in these regions are more difficult, since light can traverse the photonic crystal. Some light will be reflected at the crystal-air interface and will interfere with the light reflected from the glass-crystal interface.

In order to verify that the measured phase change is indeed due to the photonic crystal, and not simply the gold reflector, we have compared the Fabry-Pérot resonances for a glass cavity with a gold film on one side and air on the other with a resonator of the same size with air on both sides. The difference in the resonance positions stayed the same throughout the wavelength range investigated here, showing that the change in phase response presented here is only due to the photonic crystal.

The exact knowledge of the reflection phase enables us to design small-volume resonant cavities, where a small 


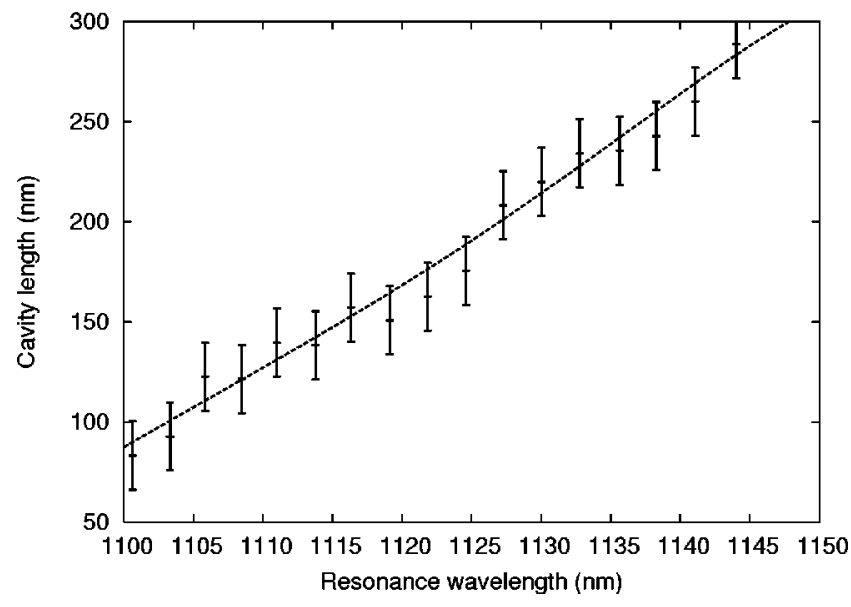

FIG. 5. Cavity size needed to meet a specified wavelength requirement. The design is implemented using the phase measurement from Fig. 4 and a cavity refractive index of 1.5 .

change in reflection phase shift has a large impact on the resonant frequency. Using the data from Fig. 4 we calculate the cavity sizes needed for a given resonance wavelength, for cavities sizes around a quarter wavelength. The results are shown in Fig. 5, for a cavity with refractive index of 1.5. For a resonance at the center of the stop band we see that the required cavity is slightly smaller than the familiar quarter wavelength employed in phase-shifted distributed feedback lasers, ${ }^{14}$ since the phase shift at the centre of the stopband is larger than $90^{\circ}$. This can be explained as follows, using a one-dimensional dielectric stack. In an ideal quarter-wave stack, which has equal optical path lengths in each material, the reflection phase at the centre of the stopband is $90^{\circ}$. When the optical path lengths in the two materials of the dielectric stack are not equal, the reflection spectrum becomes asymmetric. The reflection phase changes more quickly near one band edge than near the other one and the phase at the center of the stop band moves slightly away from $90^{\circ}$. Our colloidal crystals have a filling fraction of $74 \%$ and behave like a dielectric stack with thick high-index layers separated by thin air layers.

We can now obtain a value for the fabrication tolerances needed for a certain precision in the resonance. We fit a line with slope of $4.7 \mathrm{~nm} / \mathrm{nm}$ to the curve in Fig. 5. From this we find that, in order to obtain a resonance with a frequency precision of $2 \%$, which is a typical precision for the position of the photonic crystal stop bands, the cavity must be fabricated with a precision in its size of $4.7 \times 2 \%=9 \%$, or about $20 \mathrm{~nm}$.

In summary, we have measured the phase shift exhibited by light upon reflection from a photonic crystal. By using a very large cavity, the measurements could be done over the entire photonic crystal stop band in a single scan. We find the phase shift to vary between zero and $180^{\circ}$ across the stop band. The phase shift has a very large effect on the resonant frequencies of photonic crystal defects.

${ }^{1}$ Y. A. Vlasov, M. Deutsch, and D. J. Norris, Appl. Phys. Lett. 76, 1627 (2000).

${ }^{2}$ M. Allard, E. H. Sargent, E. Kumacheva, and O. Kalinina, Opt. Quantum Electron. 34, 27 (2002).

${ }^{3}$ Y. Akahane, T. Asano, B. Song, and S. Noda, Nature (London) 425, 944 (2003).

${ }^{4}$ K. Srinivasan, P. E. Barclay, and O. Painter, Opt. Express 12, 1458 (2004).

${ }^{5}$ S. McNab, N. Moll, and Y. Vlasov, Opt. Express 11, 2927 (2003).

${ }^{6}$ E. Istrate and E. H. Sargent, IEEE J. Quantum Electron. 41, 461 (2005).

${ }^{7}$ O. Painter and K. Srinivasan, Phys. Rev. B 68, 035110 (2003).

${ }^{8}$ E. Özbay and B. Temelkuran, Appl. Phys. Lett. 69, 743 (1996).

${ }^{9}$ E. Flück, M. Hammer, A. M. Otter, J. P. Korterik, L. Kuipers, and N. F. van Hulst, J. Lightwave Technol. 21, 1384 (2003).

${ }^{10}$ J. F. Lopez and W. L. Vos, Phys. Rev. E 66, 036616 (2002).

${ }^{11}$ D. Gerace and L. C. Andreani, Opt. Lett. 29, 1897 (2004).

${ }^{12}$ M. Allard and E. H. Sargent, Appl. Phys. Lett. 85, 5887 (2004).

${ }^{13}$ P. Jiang, J. F. Bertone, K. S. Hwang, and V. L. Colvin, Chem. Mater. 11, 2132 (1999).

${ }^{14}$ H. A. Haus and C. V. Shank, IEEE J. Quantum Electron. 12, 532 (1976). 